\title{
Disaster relief routing by considering heterogeneous vehicles and reliability of routes using an MADM approach
}

\author{
Mohammad Mahdi Nasiri* and Shadab ShisheGar
}

School of Industrial Engineering, College of Engineering, University of Tehran, Tehran, Iran

\begin{tabular}{l}
\hline C H R O N I C L E \\
\hline Article history: \\
Received December 10, 2013 \\
Received in revised format \\
16 March 2014 \\
Accepted April 292014 \\
Available online \\
May 142014 \\
\hline Keywords: \\
Relief routing \\
VRP \\
Multi-attribute decision making \\
NSGAII
\end{tabular}
A B S T R A C T

\begin{abstract}
A crucial issue in humanitarian relief operations is vehicle routing because there are many difficulties in roads that people face when a disaster is occurred. In such circumstances, selecting more reliable routes could help rescue team act more efficacious. Therefore, we propose a mathematical model that maximizes the reliability of selected routes in disaster relief routing process using a multiple attribute decision making (MADM) technique. Furthermore, the proposed model minimizes the total cost that imposed to system. For this reason, the model is a multi-objective vehicle routing problem (VRP). The problem considered here is NP-hard and its objective is to find near optimal solutions, rather than optimal ones, using the nondominated sorting genetic algorithm.
\end{abstract}

\section{Introduction}

In the past decade, we have encountered several natural disasters such as the 2004 Indian Ocean earthquake and tsunami, the 2005 Katrina hurricane in the US, the 2010 earthquake in Haiti and etc. These catastrophes leave people in unfavorable circumstances and in need of urgent medical care. In these situations, it can be necessary to supplement local capacity with regional or international aid. Generally, emergency response efforts are comprised of two stages: pre-event and post-event response. Predicting and evaluating possible dangers and developing necessary action plans for reducing damages are parts of pre-event stage while post-event response starts when the disaster is still in progress. The particular tasks at this stage are locating, allocating, organizing, routing and handling existing resources. According to the United States Comprehensive Emergency Management, four phases could be considered to manage the disaster operations: mitigation, readiness, reaction and recovery, each of which has several subsets. Our focus is on the response phase and on the emergency rescue and medical care part. In fact, we investigate the relief routing problem in disaster circumstances. In humanitarian relief operations, Vehicle Routing Problem (VRP) and supply

* Corresponding author.

E-mail address: mmnasiri@ut.ac.ir (M. M. Nasiri)

(C) 2014 Growing Science Ltd. All rights reserved. doi: $10.5267 /$ j.uscm.2014.5.004 
allocation are critically important because when a disaster occurs, relief supplies should be distributed rapidly in sufficient amounts. Due to limitations in transportation resources and relief supplies, inefficient use of resources, slow response and inadequate relief deliveries may happen. The classical vehicle routing problem minimizes total transportation costs. In humanitarian relief, one is primarily concerned with whether the routs are able to deliver the aid quickly. On the other hand, it is possible that some troubles on the way of vehicles prevent them to arrive to their destinations. As a result, an objective function could be defined to maximize travel reliability, such as the probability of vehicles for arriving to their intended destinations.

\section{Literature review}

There is a growing literature that studies relief routing problem in humanitarian logistics. We can say that the first study in this issue was done by Knott (1987) that investigated distribution of relief supplies from a distribution center to several camps by formulating an LP model with an objective function that maximizes the amount of deliveries and minimizes transportation cost. In many of studies in the literature, the problem is formulated as an integer programming problem (e.g. Tzeng et al., 2007). Recently, Torre et al. (2012) prepared a survey that reviews the problems associated with routing of vehicles within disaster-affected districts to transport goods and services to distribution points and beneficiaries. The authors categorized publications in disaster relief and humanitarian logistics by problem characteristics. For example, they classified objective functions in five categories; cost minimization, unsatisfied demand minimization, latest arrival minimization, travel reliability maximization and total response time minimization. In addition, they considered three different types for goods, which should be transferred to beneficiaries: Stochastic supply, stochastic demand and multi commodity. Routing models may differ in the number of depots: from multiple depot and single depot to no depot types. Also, they could use vehicles that have uncertain travel times or vehicles that vary in transportation capacity, speed, fuel, etc. which are called heterogeneous vehicles. By surveying the literature, it can be concluded that models with objective functions that maximizes travel reliability or minimizes arrival time, have been less studied. In addition, most of the relief routing literature focuses on pre-positioning and initial distribution of goods and services after a disaster. However, the beneficiaries may need support beyond the capacity of a single-period delivery. Consequently, the multi-period delivery routing should be considered and here is a big gap in the literature.

In relief routing models, there are several studies that their policies are based on egalitarianism (Mete $\&$ Zabinsky, 2010; Huang et al., 2012). In such papers the objective function maximizes the amount of demand satisfied or maximizes equality of a measure like delivery quantity. Mete and Zabinsky (2010) proposed stochastic optimization approach (there was uncertainty in travel time) to create a two-stage model for storage and distribution problem of supplies for the preparedness off a wide variety of disaster types. While, Huang et al. (2012) provided a paper that its goal was to study a stylized version of the last-mile delivery problem (LMDP). The LMDP designs vehicle routs and distribution schedules for a fleet of vehicles delivering supplies from a distribution center. The authors focused on a single period problem where each vehicle performs at most one trip to deliver one commodity type. Bozorgi et al. (2012) proposed a mixed-integer nonlinear programming for relief chain design problem that considered three type of uncertainty (demand, supply and cost parameters). Their proposed model minimized the sum of the expected total cost and the variance of the total cost to determine the location of relief distribution centers and the allocation of affected area to relief distribution centers.

A few papers exist in the literature that their objective functions maximize travel reliability (Ukkusuri \& Yushimito, 2008; Vitoriano et al., 2009). Hamedi et al. (2012) focused on reliability of relief transportation in disaster such that different types of vehicles are discussed. In this matter they mentioned that reliability of the route should be certain. Hence, in their study the focus is on routing 
and scheduling of trucks that transport over an unreliable road network. However, there are several depots and suppliers, the uncertainty in this problem is important. Generally, the integration of reliability and vulnerability, costs and benefits to address the uncertainty in decision making is crucial in humanitarian response. A mathematical integer programming developed to integer routing and scheduling of humanitarian trucks. A genetic algorithm used to optimize the problem. The gap of this study can be the kind of supply and demand. It means that we can consider stochastic supply and demand to develop the model and it will be more realistic.

Vehicles Routing Problem (VRP) consider models in which goods are distributed by a set of vehicles on routs beginning and ending at a single depot, multiple depot or some with no depot. Knott (1987), Huang et al. (2012), Mete and Zabinsky (2010) and Barbarosoglu and Arda (2004) consider their models by a single depot. Multiple depots exist when all vehicles have routes that originate and end from different depots.

In disaster relief operations, delivering prioritized items has an important role. Lin et al. (2011) suggested an integer programming model with multiple objectives. They tried to solve this model with two heuristic approaches: an approach based on GA and another one is developed by decomposing the original problem. Yi et al. (2006) used ant colony optimization for disaster relief operation to minimize the service delay. In their proposed method, the original emergency logistics problem is divided into two phases, i.e. the vehicle route building, and the distribution of different commodity.

Afshar and Haghani (2011) proposed a comprehensive model that controls the flow of relief commodities from sources through supply chain to hands of applicators. In this model some details such as vehicle routing, pickup and delivery schedules and optimal locations for facilities are considered. To maximize the survival rate of population and minimize the cost, the transportation of supplies should be quick and efficient. So, the objective function minimizes the total amount of weighted unsatisfied demand over all commodities, times, and demand points. This model provided the opportunity for a centralized operation plan that can eliminate delays and assign the limited resources in a way that is optimal for the entire system.

Vehicle routing problem is the area that is important after disasters. Lee (2012) worked on ambulance as a vehicle to reduce the responding time using the nearest neighborhood policy (NN). Wohlgemuth et al. (2012) also, worked on an extension of vehicle routing problem. Pickup and delivery problems are in this category, where location may both receive and send goods. The model of this paper optimizes delays and equipment utilization. Jotshi et al. (2008) developed a robust methodology for the dispatching and routing of emergency vehicles in a post-disaster environment with the support of data fusion. This study also has been tried to minimize the response time.

In the traditional vehicle routing problem (VRP), vehicles are dispatched from a single depot to service the demands of customers. In the multi-depot vehicle routing problem, which is considered by several authors, vehicles are dispatched from several depots. Gulczynski et al. (2011) developed an integer programming-based heuristic for this problem. This model was applied a heuristic to determine the decrease in distance traveled that can be achieved by allowing split deliveries among vehicles based at the same depot and vehicles based at different depots. In addition, Ho et al. (2007) proposed three hybridized heuristics: the technique of Clarke and Wright for saving, the nearest neighborhood method, and the iterated swap procedure. The major difference between the hybrid genetic algorithms (HGAs) is that the initial solutions are generated randomly in HGA1. The Clarke and Wright saving method and the nearest neighbor heuristic are incorporated into HGA2 for the initialization procedure. It was proved that the performance of HGA2 is superior to that of HGA1 in terms of the total delivery time. Furthermore, this kind of problem is studied with considering 
secondary disasters in Zhang et al. (2012). In this paper, the emergency resource allocation problem is formulated considering constraints of multiple resources and possible secondary disasters.

Considering some aspects such as reliability can make the model more effective in real world. Also, there are lots of constraints that effect on situations. In this regard, Jin Choi and Lee (2011) introduced mUSTPP-R (multiple uncapacitated symmetric traveling purchaser problem for maximizing system's reliability) to purchase the components with maximum system reliability within a given budget constraints on the basis of mUSTPP by incorporating additional constraints such as multi-purchaser, capacity, and fixed cost restrictions as a generalization of TPP.

To solve this kind of problems, we need to use different methods. Lavasani et al. (2011) presented a fuzzy Multi Attribute Decision Making (FMADM) method. This model is suitable for handling group decision making problems in a fuzzy environment. Also, it is proposed for ranking offshore well barriers from a cost-benefit view point and it can handle both fuzzy and crisp data. Finally in their study, the best well barrier or risk control option with respect to cost and benefit is selected using a Technique for Order Preference by Similarity to Ideal Solution (TOPSIS) method.

With these ideas in mind, our study tries to develop a relief routing model in disaster circumstances with considering reliability of those vehicles that are supposed to deliver commodities to beneficiaries as the objective function. In addition, we investigate the multiple depot problems where routs of vehicles begin and end at one of many designed depots. Furthermore, the kind of demand and supply will be shown in our model. Being Stochastic or deterministic for demand and supply is important in the model. According to the above literature for this problem, the model for optimizing travel cost and reliability value, with considering multi depots and effective risks can eliminate the gap of this important Issue.

\section{Problem definition}

To have a correct imagination of the problem, consider a distribution company with multiple depots that the number and location of them are predetermined. Besides, consider a disaster is occurred in the area and many geographical points are affected by this disaster and people need humanitarian aids to get survived. So, a fleet of vehicles with limited capacity is used to transport the products from depots to affected areas. Each depot has enough stock to provide all the products ordered by the people. Each vehicle starts its travel from one specific depot and finishes at the same one. The vehicles are heterogeneous which are from different types and have different capacities. The location of each affected area and its distance from the depots is known in advance. Furthermore, the amount of demand on nodes is specific. Each node is visited by a vehicle exactly once.

As mentioned earlier, this problem is a multiple-depot vehicle routing problem called MDVRP. To understand MDVRP clearly, an example with two depots and 13 areas affected by a recent earthquake is illustrated in Fig.1. The numbers above each node shows their demand. The capacity of each vehicle is equal to 12 , so they should allocate on nodes due to this constraint. A feasible solution is drawn in Fig. 1. In this study, the objective is to maximize the total reliability of selected routes and minimize the total cost of relief process. Reliability is defined as a multiple attribute decision making (MADM) concept called "graph theoretical-matrix permanent" which is help us to make decision on the best routes with the maximum reliability. This concept is explained in more details as follows:

For square matrix in linear algebra, the permanent is a function of the matrix similar to the determinant with a basic difference that in permanents the signatures of the permutations are taken as positive (Baykasoglu, 2012). As far as the entries of the matrix are concerned, the determinant as well as permanent is polynomial and both of them are special cases of the immanent, which is a more 
general function of a matrix. In mathematics, the immanent of a matrix is known to be a generalization of the concepts of determinant and permanent.

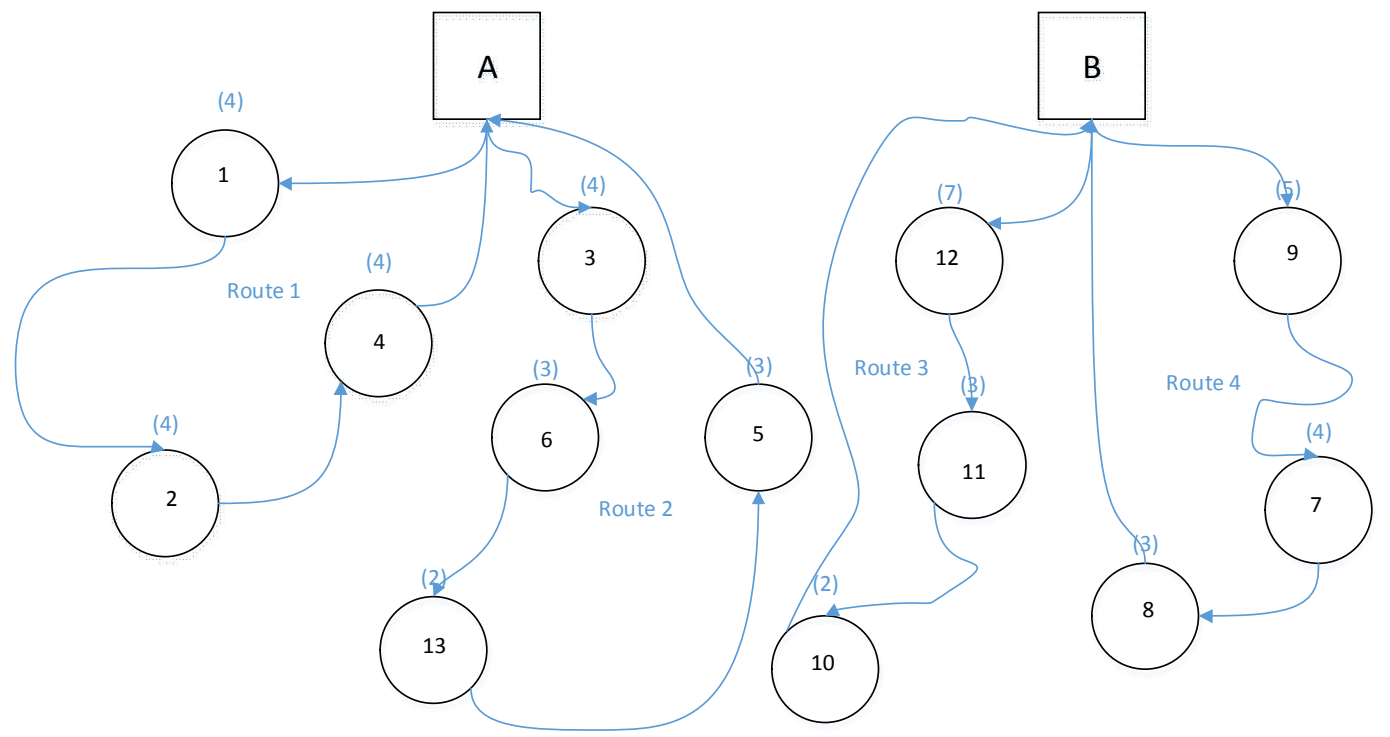

Fig. 1. A MDVRP example

Let $\lambda=\left(\lambda_{1}, \lambda_{2}, \ldots\right)$ be a partition of $\mathrm{n}$ and let $\chi_{\lambda}$ be the corresponding irreducible representationtheoretic character of the symmetric group $S_{n}$. The immanent of a $n \times n$ matrix $A=\left(a_{i j}\right)$ associated with the character $\chi_{\lambda}$ is defined as the expression:

$\operatorname{Im} m_{\lambda}(A)=\sum_{\sigma \in S_{n}} \chi_{\lambda}(\sigma) a_{1 \sigma(1)} a_{2 \sigma(2)} \ldots a_{n \sigma(n)}$

The determinant is a special case of the immanent, where $\chi_{\lambda}$ is the alternating character sgn, of $\mathrm{S}_{\mathrm{n}}$, defined by the parity of a permutation.

The permanent is the case where $\chi_{\lambda}$ is the trivial character, which is identically equal to 1 .

No information is lost in permanents, because the permutations are not taken into account (all signatures are taken as positive). Permanents are mainly used in combinatorial mathematics. Permanent defines the number of perfect matching in a two-part graph.

\subsection{Application of Graph Theory Matrix Permanent approach to Decision Making (GT-MP-DM)} problem

In this section detailed explanations about the GT-MP-DM approach in modeling and solving crisp and fuzzy MCDM problems are given.

1. Identify criteria, sub-criteria and alternatives, which are essential to MCDM problem at hand (Fig. 2).

2. Determine the "relative importance" (or interaction) between criteria and "the scores of alternatives" for each criterion. 


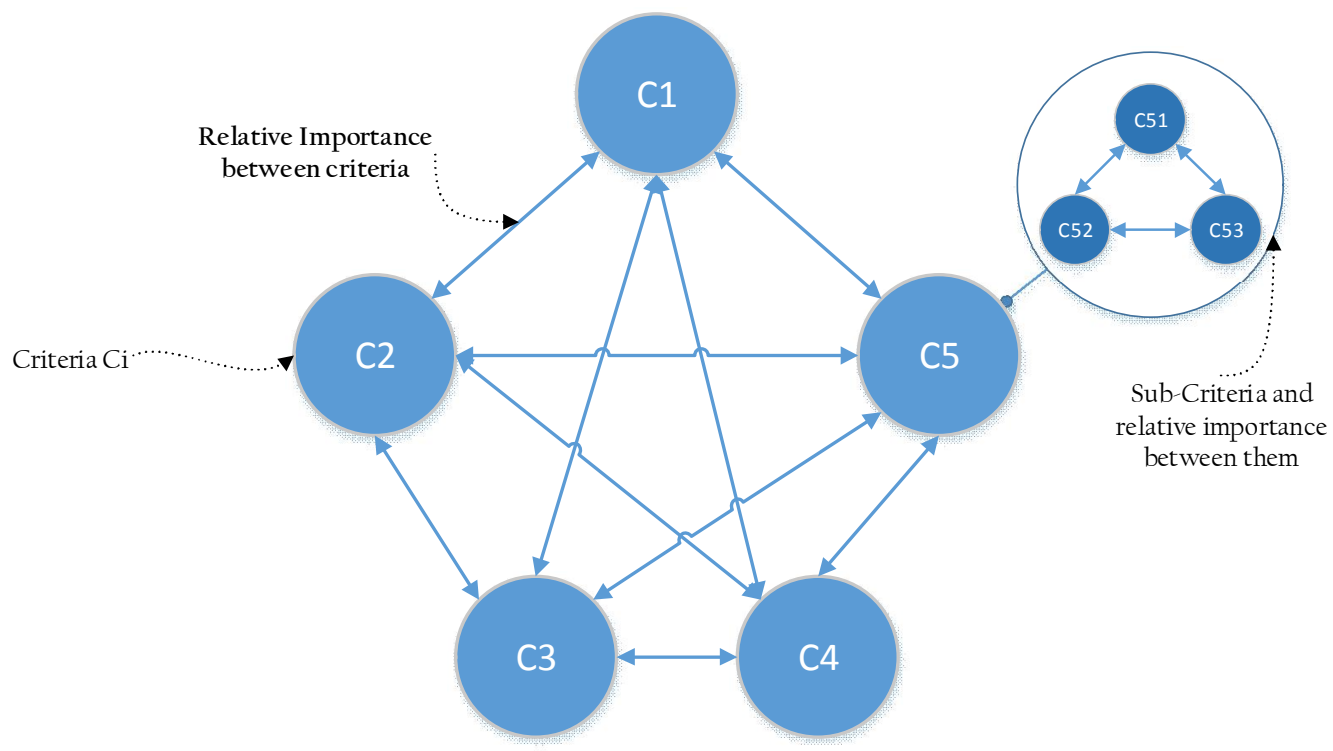

Fig. 2. Structure of MADM problem for GT-MP-DM

If the value of an alternative $C_{i}$ has a range $\left\{C_{i l}, C_{i u}\right\}$, the value 0 is assigned to lowest range $\left(C_{i l}\right)$ and 1 is assigned to highest range $\left(C_{i u}\right)$. The other intermediate values $\left(C_{i i}\right)$ can be determined by using Eq. (1) (Rao and Gandhi 2002).

$C_{i}=\left(C_{i i}-C_{i l}\right) /\left(C_{i u}-C_{i l}\right)$

For non-beneficial case the value of 0 is assigned to highest value $\left(C_{i u}\right)$ and 1 is assigned to lowest value $\left(C_{i l}\right)$. The other intermediate values can be determined by using Eq. (2).

$C_{i}=\left(C_{i u}-C_{i i}\right) /\left(C_{i u}-C_{i l}\right)$

After computing all criteria values $(i=1, \ldots, N)$ for an alternative we can define a criteria rating matrix $(\psi)$ for that alternative.

$[\psi]=\left[\begin{array}{cccc}C_{11} & 0 & \ldots & 0 \\ 0 & C_{22} & \ldots & 0 \\ . & \ldots & . & . \\ 0 & \ldots & 0 & C_{n n}\end{array}\right]$

Thus the relative importance (interaction) matrix $\beta$ as given by Eq. (4) can be symmetrical or nonsymmetrical (Garg et al., 2006).

$$
[\beta]=\left[\begin{array}{cccc}
0 & r_{12} & \ldots & r_{1 n} \\
r_{21} & 0 & \ldots & r_{2 n} \\
. & \ldots & . & . \\
r_{n 1} & \ldots & \ldots & 0
\end{array}\right]
$$


3. After obtaining criteria rating matrix $(\psi)$ for an alternative and relative importance (interaction) matrix $\beta$ between criteria the next step is to form alternative evaluation matrix ( $\xi$ ) which is given by Eq. (5).

$$
\xi=\psi+\beta=\left[\begin{array}{ccccc}
C_{1} & r_{12} & r_{13} & \ldots & r_{1 n} \\
r_{21} & C_{2} & r_{23} & \ldots & r_{2 n} \\
r_{31} & r_{32} & C_{3} & \ldots & r_{3 n} \\
\ldots & \ldots & \ldots & \ldots & \ldots \\
r_{n 1} & r_{n 2} & r_{n 3} & \ldots & C_{n}
\end{array}\right]
$$

The permanent of matrix $\xi \operatorname{per}(\xi)$ gives the rating for the alternative. For each alternative $\operatorname{per}(\xi)$ must be calculated and arranged in a descending order. The alternative with the highest $\operatorname{per}(\xi)$ value is the best alternative. $\operatorname{per}(\xi)$ can be calculated as explained in the previous section by using Eq. 3 . An expanded version of this equation is given in Eq. (6).

$$
\begin{aligned}
& \operatorname{per}(\xi)=\prod_{i=1}^{N} C_{i}+\sum_{i, j, \ldots, N}\left(r_{i j} r_{j i}\right) C_{k} C_{l} \ldots C_{N}+\sum_{i, j, \ldots, N}\left(r_{i j} r_{j k} r_{k i}+r_{i k} r_{k j} r_{j i}\right) C_{l} C_{n} \ldots C_{N} \\
& +\left\{\sum_{i, j, \ldots, N}\left(r_{i j} r j i\right)\left(r_{k l} r_{l k}\right) C_{n} C_{m} \ldots C_{N}+\sum_{i, j, \ldots, N}\left(r_{i j} r_{j k} r_{k l} r_{l i}+r_{i l} r_{l k} r_{k j} r_{j i}\right) C_{n} C_{m} \ldots C_{N}\right\} \\
& +\left[\sum_{i, j, \ldots, N}\left(r_{i j} r_{j i}\right)\left(r_{k l} r_{\ln } r_{n k}+r_{k n} r_{n l} r_{l k}\right) C_{m} C_{0} \ldots C_{N}+\sum_{i, j, \ldots, N}\left(r_{i j} r_{j k} r_{k l} r_{\mathrm{ln}} r_{l i}+r_{i n} r_{n l} r_{l k} r_{l j} r_{j i}\right) C_{m} C_{0} \ldots C_{N}\right]+\ldots
\end{aligned}
$$

With the permanent obtained from these equations, we can rate alternatives. Each alternative has more permanent value is better for using (Baykasoglu, 2012).

\subsection{Application of GT-MP-DM to relief routing problem}

In this section detailed explanations about GT-MP-DM approach in relief routing modeling are given. The methodology explained in a step by step manner in order to bring a better understanding (Baykasoglu, 2012).

Step-1: Identify criteria that influence the route reliability. By surveying several disaster and other geoscience databases like the International Disaster Database, Earthquake Database of Australia and Canadian Disaster Database, significant effective factors on reliability of routes in earthquakes are produced. According to this disquisition, the road type, geographical characteristics, mountainous rate of the road and its length from depot to affected areas effect the reliability of routes when an earthquake is occurred. So, matrix $\beta$ could be defined as below:

Step-2: Determination of the matrix $\beta$ which its elements represent the "relative importance" between criteria. Here for instance $a b=0.2$ and $b a=0.8$ means that criteria $\mathrm{b}$ is very important than criteria a.

$a$ : Road type(autobahn, arterial, lane, highway, etc.)

$b$ : Geographical characteristics

$c$ : Distance

$d$ : Mountainous rate 


$$
\beta=\left(\begin{array}{llll}
a a & a b & a c & a d \\
b a & b b & b c & b d \\
c a & c b & c c & c d \\
d a & d b & d c & d d
\end{array}\right)=\left(\begin{array}{cccc}
0 & 0.2 & 0.4 & 0.1 \\
0.8 & 0 & 1 & 0.5 \\
0.6 & 0 & 0 & 0.3 \\
0.9 & 0.5 & 0.7 & 0
\end{array}\right)
$$

Then for each route $i-j$ "the scores of alternatives" for each criterion is investigated. For instance the $\Psi$ matrix for route 3-6 is:

$$
\Psi_{3-6}=\left(\begin{array}{cccc}
0.7 & 0 & 0 & 0 \\
0 & 0.1 & 0 & 0 \\
0 & 0 & 0.9 & 0 \\
0 & 0 & 0 & 0.6
\end{array}\right)
$$

Step-3: For calculating the permanent of the route 3-6, do as below:

$$
\xi_{3-6}=\Psi_{3-6}+\beta=\left(\begin{array}{cccc}
0.7 & 0.2 & 0.4 & 0.1 \\
0.8 & 0.1 & 1 & 0.5 \\
0.6 & 0 & 0.9 & 0.3 \\
0.9 & 0.5 & 0.7 & 0.6
\end{array}\right) \longrightarrow \operatorname{permanent}\left(\xi_{3-6}\right)=0.3573
$$

Other route permanents are calculated as above.

\section{Mathematical programming model}

Our proposed model is considered some assumptions:

- The network is discrete where the depots and affected areas are located on the nodes

- The location of the depots are known

- Depots have enough inventory to supply beneficiaries

- The capacity of each vehicle is specific

- The location of affected points toward depots is known

- The amount of demand for each node is specific and constant

\subsection{Notation and terminology}

Parameters:

$n:$ The number of affected areas in the disaster indexed $i, i \in\{1,2, \ldots, n\}$

$m$ : The number of depots indexed $i, i \in\{n+1, \ldots, m\}$

$m+n$ : The number of total nodes

$d_{i}$ : The demand of node $i$

$V:$ The number of vehicle types indexed $v, v \in\{1,2, \ldots, V\}$

$q_{v}:$ The capacity of the vehicle type $v$

$C_{v}:$ The fixed cost of the vehicle type $v$

$c_{v}$ : The cost of one unit length moving along a route 
$f_{i j}$ : The cost of one unit load on the route $i-j$

$r_{i j}$ : The distance between node $i$ and $j$

$P$ : Maximum number of nodes that each vehicle allowed to visit

Decision variables:

$x_{i j v d}: 1 \quad$ if the vehicle type $v$ starts its travel from node $d$ and continues along the route $i$ and $j$

0 Otherwise

$y_{i j}$ : is a non-negative integer variable denoting the total load remaining in the vehicle before reaching node $j$ while travelling along the route $i-j$

$u_{i v}$ : is an auxiliary and sequential variable that shows the number of node that vehicle $v$ is visiting and used in sub-tour elimination constraints.

\subsection{Formulation}

The formulation of the mathematical model is as follows.

$$
\begin{array}{cr}
\max \sum_{i=1}^{m+n-1} \sum_{j=1}^{n} \text { permanent }_{i j} x_{i j v d} & \forall v=1,2, \ldots, V \\
\min \sum_{i=1}^{m+n-1} \sum_{j=1}^{n} \sum_{v=1}^{V} \sum_{d=n+1}^{n+m} C_{v} x_{i j v d}+\sum_{i=1}^{m+n} \sum_{j=1}^{n} f_{i j} y_{i j}+\sum_{i=1}^{m+n-1} \sum_{j=1}^{n} \sum_{v=1}^{V} \sum_{d=n+1}^{n+m} c_{v} r_{i j} x_{i j v d}
\end{array}
$$

Subject to

$$
\begin{array}{lc}
\sum_{i=1}^{n+m} \sum_{v=1}^{V} \sum_{d=n+1}^{n+m} x_{i j v d}=1 & \forall j=1, \ldots n \\
\sum_{j=1}^{n+m} \sum_{v=1}^{V} \sum_{d=n+1}^{n+m} x_{i j v d}=1 & \forall i=1, \ldots, n \\
\sum_{i=1}^{n+m} x_{i j v d}-\sum_{i=1}^{n+m} x_{j v d d}=0 & \forall v=1, \ldots, V \\
\sum_{i=n+1}^{n+m} \sum_{j=1}^{n} y_{i j}=\sum_{j=1}^{n} d_{j} & \forall j=1, \ldots n+m \\
\sum_{i=1}^{n+m} y_{i j}-\sum_{i=1}^{n+m} y_{j i}=d_{j} & \forall d=n+1, \ldots, n+m \\
y_{i j} \leq \sum_{d=n+1}^{n+m} \sum_{v=1}^{V} q_{v} x_{i j v d} & \forall j=1, \ldots, n \\
& \forall i=1, \ldots, n+m \\
u_{i v}-u_{j v}+x_{i j v d}\left(q_{v}-d_{i}\right) \leq q_{v} & \forall j=1, \ldots, n \\
& \forall i=1, \ldots, n+m \\
& \forall j=1, \ldots, n \\
x_{d_{i} v d_{2}}=0 & \forall v=1, \ldots, V \\
& \forall d=n+1, \ldots, m+n \\
& \forall i=1, \ldots, n \\
& \forall v=1, \ldots, V \\
& \forall d_{1} \neq d_{2}=n+1, \ldots, m+n
\end{array}
$$




$$
\begin{array}{ll} 
& \forall i=1, \ldots, n \\
x_{i d_{1} v d_{2}}=0 & \forall v=1, \ldots, V \\
& \forall d_{1} \neq d_{2}=n+1, \ldots, m+n \\
& \forall i, j=1, \ldots, n+m \\
x_{i j v d} \in\{0,1\} & \forall v=1, \ldots, V \\
y_{i j} \geq 0, \text { int } & \forall d=n+1, \ldots, m+n \\
u_{i v} \in\{1,2, \ldots, p\} & \forall i, j=1, \ldots, m+n \\
& \forall i=1, \ldots, n+m \\
& \forall v=1, \ldots, V
\end{array}
$$

The first objective function (7) maximizes reliability of routes through maximizing summation of permanent for each route. The second objective function (8) describes the total cost that includes both the vehicle fixed cost and the travelling cost. Constraint (9) and (10) ensure that each affected point is visited only once by one vehicle. Constraint (11) illustrates that when a vehicle enters an affected node to aid people there, it must leave that node. Constraint (12) ensures that the total quantity of all vehicles that leave the depots is exactly equal to the total demand and (13) guarantees that the quantity of relief goods remaining after visiting node $\mathrm{j}$ is exactly the load before visiting this node minus its demand. Constraint (14) makes certain that the amount of relief good remains in vehicle $\mathrm{v}$ is less than its capacity. Constraint (15) prevents model creating subtours (subtour elimination). Constraints (16) and (17) ensure that when a vehicle leaves a depot, it could go to another depot and the depots only could be linked to affected areas. Constraint (18) refers to the binary of the $x_{i j v d}$ and Eq. (19) to the non-negativity of the $y_{i j}$ and (20) to the sequential of the $u_{i v}$ respectively.

\section{Solution methodology}

To solve the mathematical model, we apply the well-known method for multi-objective programming models NSGAII (Non-dominated Storing Genetic Algorithm II) which is a popular non-domination based genetic algorithm. Simulation results on different test problems show that NSGAII is able to find a much better solutions and better convergence near the true Pareto-optimal front compared to Pareto-archived evolution strategy. Figure 3, shows the flowchart of NSGAII.

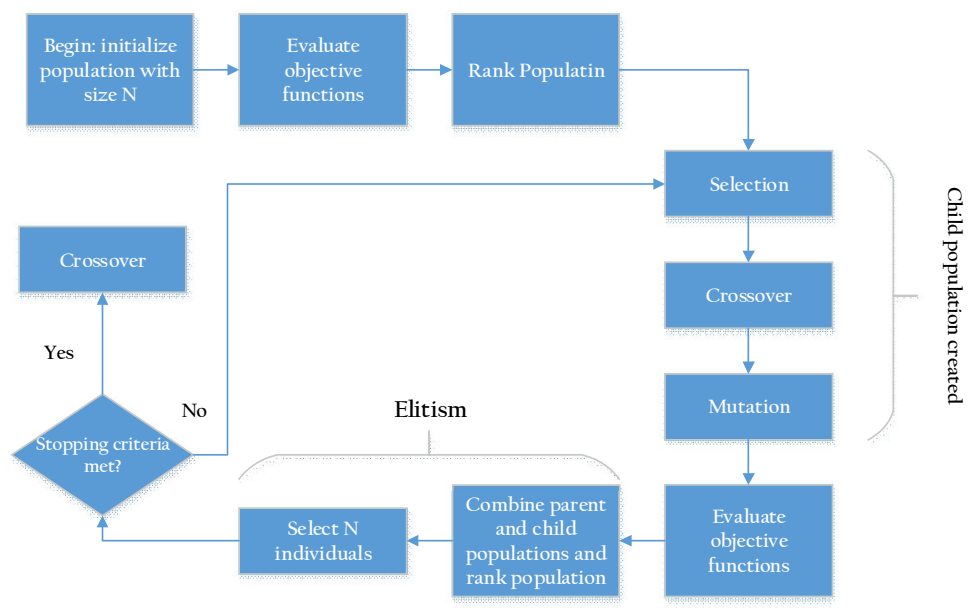

Fig. 3. Pseudo-Code of NSGAII

As usual, the population is initialized and sorted based on non-dominant into each front. The first front is completely non-dominant set in the current population and the second front being dominated by the individuals in the first front only and the front goes on. Each individual is assigned fitness 
values. Then parents are selected from the population by using binary tournament selection based on the rank. The selected population generates offsprings from crossover and mutation operators. The new generation is evaluated by objective function value and sorted again based on non-domination and only $\mathrm{N}$ individuals are selected. Finally, the stopping criterion is checked. If it is satisfied, the near optimal solution is achieved. Otherwise, return to step 4.

\subsection{Numerical experiments}

In this part firstly, a small-sized test problem with 5 affected area and 2 depots is solved using GAMS 24.0.2 software. The data of both small-sized (Table 1, 2 and 3) and large-sized (Table 4, 5 and 6) test problems obtained from "the international disaster database" website and some other disaster databases by using some satellite maps.

Table 1

Demand of affected area $i$ and depot $j$

\begin{tabular}{cccccc|cc}
\hline & \multicolumn{9}{c}{$\mathrm{n}$} & \multicolumn{3}{c}{$\mathrm{M}$} \\
\cline { 2 - 8 }$d_{i}$ & 1 & 2 & 3 & 4 & 5 & 6 & 7 \\
\hline
\end{tabular}

Table 2

Maximum capacity, fixed cost and the cost of one unit move for vehicle $v$

\begin{tabular}{cccc}
\hline & & $v$ & \\
\cline { 2 - 4 } & 1 & 47 & \\
\hline$q_{v}$ & 60 & 190 & \\
$C_{v}$ & 210 & 53 \\
\hline$c_{v}$ & 50 & 5 \\
\hline
\end{tabular}

Table 3

Distance between nodes, cost of one unit load and the permanent of the route $i-j$

\begin{tabular}{|c|c|c|c|c|c|c|c|c|c|c|c|c|c|c|c|c|c|c|c|c|c|}
\hline \multirow[b]{2}{*}{ Node } & \multicolumn{7}{|c|}{$r_{i j}$} & \multicolumn{7}{|c|}{$f_{i j}$} & \multicolumn{7}{|c|}{ permanent $_{i j}$} \\
\hline & 1 & 2 & 3 & 4 & 5 & 6 & 7 & 1 & 2 & 3 & 4 & 5 & 6 & 7 & 1 & 2 & 3 & 4 & 5 & 6 & 7 \\
\hline 1 & 0 & 8 & 15.2 & 12 & 13.6 & 5 & 10 & 0 & 1 & 2 & 5 & 5 & 1 & 3 & 0 & .93 & .45 & .81 & .43 & .87 & .6 \\
\hline 2 & 8 & 0 & 11.1 & 2 & 7 & 10 & 14.3 & 1 & 0 & 4 & 2 & 6 & 4 & 6 & .93 & 0 & .23 & .87 & .13 & .61 & .61 \\
\hline 3 & 15.2 & 11.1 & 0 & 10 & 3 & 13 & 6.2 & 2 & 4 & 0 & 3 & 2 & 4 & 3 & .45 & .23 & 0 & .19 & .79 & .53 & .92 \\
\hline 4 & 12 & 2 & 10 & 0 & 2 & 5 & 7 & 5 & 2 & 3 & 0 & 6 & 5 & 5 & .81 & .87 & .19 & 0 & .41 & .89 & .61 \\
\hline 5 & 13.6 & 7 & 3 & 2 & 0 & 8.3 & 4 & 5 & 6 & 2 & 6 & 0 & 3 & 2 & .43 & .13 & .79 & .41 & 0 & .64 & .79 \\
\hline
\end{tabular}

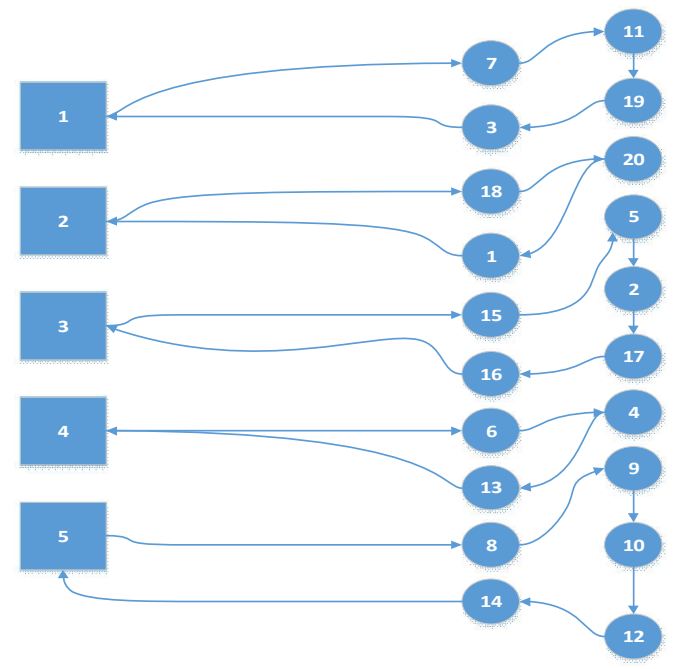

Fig. 4. Schematic result for large-sized test problem 
Small-sized test problem results, which obtained from the GAMS 24.0.2 are 6.06 for the first objective function and 1412 for the second one. Now for solving medium and large-sized problem we consider a random instance generator, which is designed to produce numerical instances for the computational experiments. This tool that is suggested Lin et al. (2011) generates the location of the depots and all nodes randomly from their corresponding ranges (Tables 4-6).

Table 4

Data for large-sized problem

\begin{tabular}{|c|c|c|c|c|c|c|c|c|c|c|c|c|c|c|c|c|c|c|c|c|c|c|c|c|c|}
\hline \multirow{2}{*}{$r_{i j}$} & \multicolumn{20}{|c|}{ Affected Area } & \multicolumn{5}{|c|}{ Depot } \\
\hline & 1 & 2 & 3 & 4 & 5 & 6 & 7 & 8 & 9 & 10 & 11 & 12 & 13 & 14 & 15 & 16 & 17 & 18 & 19 & 20 & 21 & 22 & 23 & 24 & 25 \\
\hline 1 & 0 & 13 & 11 & 14 & 16 & 11 & 15 & 19 & 18 & 12 & 13 & 11 & 17 & 10 & 13 & 12 & 17 & 14 & 11 & 3 & 10 & 2 & 15 & 23 & 27 \\
\hline 2 & 13 & 0 & 21 & 10 & 3 & 10 & 11 & 13 & 20 & 14 & 12 & 17 & 20 & 19 & 12 & 16 & 4 & 10 & 14 & 20 & 23 & 11 & 17 & 16 & 20 \\
\hline 3 & 11 & 21 & 0 & 21 & 20 & 12 & 11 & 11 & 19 & 20 & 17 & 20 & 21 & 13 & 19 & 22 & 18 & 21 & 8 & 21 & 3 & 13 & 21 & 7 & 12 \\
\hline 4 & 14 & 10 & 21 & 0 & 18 & 1 & 18 & 9 & 20 & 17 & 18 & 14 & 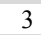 & 13 & 9 & 16 & 16 & 20 & 11 & 20 & 10 & 23 & 19 & 11 & 15 \\
\hline 5 & 16 & 3 & 20 & 18 & 0 & 16 & 10 & 13 & 19 & 13 & 14 & 10 & 18 & 16 & 7 & 14 & 11 & 9 & 17 & 18 & 17 & 27 & 7 & 14 & 13 \\
\hline 6 & 11 & 10 & 12 & 1 & 16 & 0 & 22 & 12 & 14 & 15 & 20 & 21 & 16 & 20 & 13 & 16 & 13 & 15 & 11 & 15 & 23 & 19 & 9 & 1 & 12 \\
\hline 7 & 15 & 11 & 11 & 18 & 10 & 22 & 0 & 14 & 18 & 17 & 5 & 18 & 12 & 11 & 15 & 10 & 16 & 14 & 12 & 11 & 1 & 14 & 18 & 21 & 17 \\
\hline 8 & 19 & 13 & 11 & 9 & 13 & 12 & 14 & 0 & 1 & 15 & 15 & 17 & 20 & 17 & 20 & 20 & 18 & 14 & 10 & 9 & 19 & 14 & 20 & 13 & 3 \\
\hline 9 & 18 & 20 & 19 & 20 & 19 & 14 & 18 & 1 & 0 & 6 & 10 & 19 & 10 & 13 & 17 & 15 & 13 & 18 & 20 & 16 & 19 & 18 & 17 & 15 & 4 \\
\hline 10 & 12 & 14 & 20 & 17 & 13 & 15 & 17 & 15 & 0 & 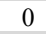 & 12 & 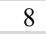 & 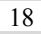 & 11 & 16 & 13 & 18 & 19 & 11 & 19 & 18 & 19 & 16 & 16 & 10 \\
\hline 11 & 13 & 12 & 17 & 18 & 14 & 20 & 5 & 15 & 10 & 12 & 0 & 15 & 13 & 10 & 16 & 9 & 11 & 19 & 7 & 18 & 6 & 19 & 16 & 21 & 18 \\
\hline 12 & 11 & 17 & 20 & 14 & 10 & 21 & 18 & 17 & 19 & 8 & 15 & 0 & 18 & 1 & 11 & 13 & 18 & 1 & 15 & 16 & 19 & 18 & 11 & 22 & 4 \\
\hline 13 & 17 & 20 & 21 & 3 & 18 & 16 & 12 & 20 & 10 & 18 & 13 & 18 & 0 & 19 & 13 & 13 & 18 & 14 & 20 & 16 & 13 & 14 & 13 & 3 & 22 \\
\hline 14 & 10 & 19 & 13 & 13 & 16 & 20 & 11 & 17 & 13 & 11 & 10 & 1 & 19 & 0 & 18 & 10 & 15 & 11 & 13 & 13 & 12 & 11 & 10 & 1 & 3 \\
\hline 15 & 13 & 2 & 19 & 9 & 7 & 13 & 15 & 20 & 17 & 16 & 16 & 11 & 13 & 18 & 0 & 13 & 18 & 18 & 12 & 17 & 16 & 18 & 0 & 14 & 21 \\
\hline 16 & 12 & 16 & . & 19 & 1 & 16 & 10 & 20 & 15 & 13 & (1) & 13 & 13 & 10 & 13 & 10 & . & 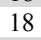 & 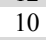 & 11 & 11 & (20 & 2 & 14 & 13 \\
\hline 17 & 17 & 4 & 18 & 16 & 11 & 13 & 16 & 18 & 13 & 18 & 11 & 18 & 18 & 15 & 18 & 2 & 0 & 12 & 13 & 19 & 17 & 19 & 4 & 14 & 18 \\
\hline 18 & 14 & 10 & 21 & 20 & 9 & 15 & 14 & 14 & 18 & 19 & 19 & 18 & 14 & 11 & 18 & 18 & 21 & 0 & 19 & 1 & 15 & 0 & 18 & 16 & 14 \\
\hline 19 & 11 & 14 & $\gamma$ & 11 & 17 & 11 & 12 & 10 & 20 & 11 & 7 & 15 & 20 & 13 & 12 & 10 & 13 & 19 & 0 & 17 & 11 & 13 & 13 & 16 & 16 \\
\hline 20 & 3 & 20 & 21 & 20 & 18 & 15 & 11 & 9 & 16 & 19 & 18 & 16 & 16 & 13 & 17 & 11 & 19 & 1 & 17 & 0 & 12 & 1 & 13 & 16 & 12 \\
\hline
\end{tabular}

Table 5

Data for large-sized problem

\begin{tabular}{|c|c|c|c|c|c|c|c|c|c|c|c|c|c|c|c|c|c|c|c|c|c|c|c|c|c|}
\hline$f_{i j}$ & \multicolumn{20}{|c|}{ Affected Area } & \multicolumn{5}{|c|}{ Depot } \\
\hline 1 & 0 & 3 & 3 & 8 & 7 & 3 & 8 & 4 & 3 & 8 & 7 & 8 & 6 & 4 & 5 & 8 & 7 & 6 & 5 & 1 & 5 & 1 & 6 & 8 & 4 \\
\hline 2 & 3 & 0 & 5 & 5 & 1 & 4 & 3 & 3 & 8 & 8 & 6 & 4 & 7 & 8 & 6 & 3 & 2 & 4 & 7 & 4 & 5 & 8 & 2 & 5 & 6 \\
\hline 3 & 3 & 5 & 0 & 6 & 6 & 8 & 7 & 4 & 6 & 4 & 6 & 5 & 3 & 6 & 3 & 6 & 5 & 3 & 1 & 3 & 1 & 7 & 1 & 4 & 5 \\
\hline 4 & 8 & 5 & 6 & 0 & 3 & 2 & 7 & 4 & 4 & 8 & 7 & 3 & 1 & 5 & 3 & 6 & 3 & 6 & 7 & 3 & 7 & 4 & 6 & 4 & 5 \\
\hline 5 & 7 & 1 & 6 & 3 & 0 & 9 & 6 & 7 & 4 & 9 & 8 & 7 & 8 & 7 & 2 & 7 & 5 & 5 & 8 & 4 & 3 & 4 & 7 & 4 & 6 \\
\hline 6 & 3 & 4 & 8 & 2 & 9 & 0 & 6 & 7 & 8 & 4 & 6 & 8 & 6 & 5 & 7 & 4 & 4 & 4 & 8 & 4 & 5 & 4 & 6 & 1 & 7 \\
\hline 7 & 8 & 3 & 7 & 7 & 6 & 6 & 0 & 7 & 6 & 6 & 1 & 7 & 3 & 6 & 7 & 6 & 6 & 8 & 6 & 8 & 2 & 8 & 5 & 6 & 5 \\
\hline 8 & 4 & 3 & 4 & 4 & 7 & 7 & 7 & 0 & 2 & 7 & 5 & 6 & 6 & 4 & 3 & 3 & 8 & 4 & 5 & 4 & 5 & 5 & 2 & 7 & 1 \\
\hline 9 & 3 & 8 & 6 & 4 & 4 & 8 & 6 & 2 & 0 & 1 & 3 & 3 & 5 & 6 & 5 & 7 & 7 & 5 & 7 & 3 & 3 & 4 & 4 & 3 & 6 \\
\hline 10 & 8 & 8 & 4 & 8 & 9 & 4 & 6 & 7 & 1 & 0 & 8 & 2 & 8 & 7 & 8 & 6 & 7 & 7 & 6 & 6 & 2 & 7 & 5 & 8 & 4 \\
\hline 11 & 7 & 6 & 6 & 7 & 8 & 6 & 1 & 5 & 3 & 8 & 0 & 7 & 5 & 6 & 4 & 5 & 7 & 5 & 2 & 7 & 5 & 6 & 6 & 6 & 3 \\
\hline 12 & 8 & 4 & 5 & 3 & 7 & 8 & 7 & 6 & 3 & 2 & 7 & 0 & 7 & 1 & 3 & 7 & 7 & 3 & 5 & 6 & 3 & 7 & 6 & 4 & 4 \\
\hline 13 & 6 & 7 & 3 & 1 & 8 & 6 & 3 & 6 & 5 & 8 & 5 & 7 & 0 & 7 & 6 & 5 & 4 & 6 & 8 & 4 & 4 & 5 & 5 & 1 & 5 \\
\hline 14 & 4 & 8 & 6 & 5 & 7 & 5 & 6 & 4 & 6 & 7 & 6 & 1 & 7 & 0 & 4 & 7 & 4 & 6 & 5 & 7 & 2 & 5 & 3 & 4 & 2 \\
\hline 15 & 5 & 6 & 3 & 3 & 2 & 7 & 7 & 3 & 5 & 8 & 4 & 3 & 6 & 4 & 0 & 7 & 3 & 7 & 8 & 5 & 4 & 8 & 1 & 5 & 6 \\
\hline 16 & 8 & 3 & 6 & 6 & 7 & 4 & 6 & 3 & 7 & 6 & 5 & 7 & 5 & 7 & 7 & 0 & 2 & 5 & 6 & 5 & 6 & 3 & 2 & 4 & 4 \\
\hline 17 & 7 & 2 & 5 & 3 & 5 & 4 & 6 & 8 & 7 & 7 & 7 & 7 & 4 & 4 & 3 & 2 & 0 & 6 & 5 & 4 & 7 & 3 & 4 & 7 & 6 \\
\hline 18 & 6 & 4 & 3 & 6 & 5 & 4 & 8 & 4 & 5 & 7 & 5 & 3 & 6 & 6 & 7 & 5 & 6 & 0 & 7 & 1 & 7 & 1 & 3 & 3 & 2 \\
\hline 19 & 5 & 7 & 1 & 7 & 8 & 8 & 6 & 5 & 7 & 6 & 2 & 5 & 8 & 5 & 8 & 6 & 5 & 7 & 0 & 5 & 4 & 4 & 5 & 3 & 3 \\
\hline 20 & 1 & 4 & 3 & 3 & 4 & 4 & 8 & 4 & 3 & 6 & 7 & 6 & 4 & 7 & 5 & 5 & 4 & 1 & 5 & 0 & 2 & 5 & 5 & 4 & 4 \\
\hline
\end{tabular}

The objective function value of this test problem is obtained by using MATLAB R2012a, which is 21.286 for the first objective function and 2876 for the second one. Schematic result of this test problem is shown at Fig. 4. 
Table 6

Data for large-sized problem

\begin{tabular}{|c|c|c|c|c|c|c|c|c|c|c|c|c|c|c|c|c|c|c|c|c|c|c|c|c|c|}
\hline$p e r_{i j}$ & & & & & & & & & & Affec & ed Area & & & & & & & & & & & & Depot & & \\
\hline 1 & 0 & .24 & .047 & .634 & .115 & .02 & .201 & .295 & .266 & .063 & .748 & .844 & .027 & .413 & .543 & .413 & .082 & .471 & .112 & .978 & .281 & .634 & .267 & .156 & .276 \\
\hline 2 & .24 & 0 & .460 & .169 & .891 & .096 & .154 & .052 & .724 & .281 & .333 & .588 & .015 & .222 & .415 & .051 & .795 & .705 & .872 & .182 & .356 & .324 & .678 & .287 & .45 \\
\hline 3 & .047 & .460 & 0 & .036 & .240 & .263 & .379 & .733 & .437 & .377 & .905 & .169 & .485 & .646 & .866 & .713 & .807 & .105 & .986 & .659 & .935 & .857 & .394 & .253 & .398 \\
\hline 4 & .634 & .169 & .036 & 0 & .618 & .805 & .662 & .122 & .045 & .141 & .116 & .278 & .904 & .079 & .466 & .281 & .347 & .693 & .626 & 0.147 & .845 & .746 & .485 & .268 & .293 \\
\hline 5 & .115 & .891 & .240 & .618 & 0 & .087 & .523 & .86 & .139 & .119 & .09 & .013 & .551 & .739 & .923 & .093 & .003 & .123 & .795 & .764 & .273 & .387 & .926 & .384 & .736 \\
\hline 6 & .02 & .096 & .263 & .805 & .087 & 0 & .706 & .259 & .323 & .103 & .395 & .773 & .288 & .118 & .79 & .49 & .392 & .746 & .556 & .006 & .274 & .638 & .728 & .994 & .362 \\
\hline 7 & .201 & .154 & .379 & .662 & .523 & .706 & 0 & .608 & .73 & .525 & .97 & .124 & .052 & .523 & .415 & .557 & .525 & .53 & .872 & .745 & .932 & .273 & .236 & .482 & .73 \\
\hline 8 & .295 & .052 & .733 & .122 & .860 & .259 & .608 & 0 & .907 & .468 & .165 & .195 & .293 & .044 & .545 & .038 & .848 & .64 & .142 & .026 & .384 & .473 & .285 & .264 & .899 \\
\hline 9 & .266 & .724 & .437 & .045 & .139 & .323 & .730 & .907 & 0 & .924 & .849 & .721 & .876 & .991 & .497 & .394 & .161 & .57 & .295 & .201 & .374 & .384 & .472 & .472 & .578 \\
\hline 10 & .063 & .281 & .377 & .141 & .119 & .103 & .525 & .468 & .924 & 0 & .174 & .934 & .479 & .543 & .203 & .714 & .315 & .347 & .288 & .055 & .376 & .372 & .594 & .732 & .127 \\
\hline 11 & .748 & .333 & .905 & .116 & .090 & .395 & .970 & .165 & .849 & .174 & 0 & .637 & .480 & .817 & .060 & .825 & .564 & .153 & .940 & .415 & .733 & .152 & .373 & .162 & .199 \\
\hline 12 & .844 & .588 & .169 & .278 & .013 & .773 & .124 & .195 & .721 & .934 & .637 & 0 & .216 & .992 & .382 & .573 & .131 & .053 & .297 & .858 & .376 & .126 & .16 & .172 & .374 \\
\hline 13 & .027 & .015 & .485 & .904 & .551 & .288 & .052 & .293 & .876 & .479 & .480 & .216 & 0 & .113 & .160 & .179 & .773 & .036 & .692 & .58 & .362 & .376 & .472 & .892 & .53 \\
\hline 14 & .413 & .222 & .646 & .079 & .739 & .118 & .523 & .044 & .991 & .543 & .817 & .992 & .113 & 0 & .818 & .536 & .149 & .793 & .667 & .284 & .142 & .189 & .901 & .326 & .935 \\
\hline 15 & .543 & .415 & .866 & .466 & .923 & .790 & .415 & .545 & .497 & .203 & .060 & .382 & .160 & .818 & 0 & .042 & .282 & .737 & .115 & .138 & .376 & .47 & .982 & .365 & .173 \\
\hline 16 & .413 & .051 & .713 & .281 & .093 & .490 & .557 & .038 & .394 & .714 & .825 & .573 & .179 & .536 & .042 & 0 & .964 & .173 & .743 & .178 & .236 & .473 & .945 & .237 & .69 \\
\hline 17 & .082 & .795 & .807 & .347 & .003 & .392 & .525 & .848 & .161 & .315 & .564 & .131 & .773 & .149 & .282 & .964 & 0 & .642 & .768 & .55 & .574 & .472 & .84 & .263 & .491 \\
\hline 18 & .471 & .705 & .105 & .693 & .123 & .746 & .530 & .640 & .570 & .347 & .153 & .053 & .036 & .793 & .737 & .173 & .642 & 0 & .224 & .969 & .372 & .927 & .572 & .72 & .592 \\
\hline 19 & .112 & .872 & .986 & .626 & .795 & .556 & .872 & .142 & .295 & .288 & .940 & .297 & .692 & .667 & .115 & .743 & .768 & .224 & 0 & .105 & .832 & .129 & .47 & .379 & .25 \\
\hline 20 & .978 & .182 & .659 & .147 & .764 & .006 & .745 & .026 & .201 & .055 & .415 & .858 & .580 & .284 & .138 & .178 & .550 & .969 & .105 & 0 & .473 & .042 & .371 & .192 & .371 \\
\hline \multirow{5}{*}{$d_{j}$} & 1 & 2 & 3 & 4 & 5 & 6 & 7 & 8 & 9 & 10 & 11 & 12 & 13 & 14 & 15 & 16 & 17 & 18 & 19 & 20 & & & $\mathrm{v}$ & & \\
\hline & 10 & 4 & 17 & 10 & 6 & 20 & 20 & 25 & 25 & 10 & 13 & 15 & 10 & 5 & 16 & 13 & 20 & 25 & 19 & 30 & 1 & 2 & 3 & 4 & 5 \\
\hline & & & & & & & & & & & & & & & & & & & & $\overline{C_{v}}$ & 220 & 200 & 210 & 230 & 200 \\
\hline & & & & & & & & & & & & & & & & & & & & $q_{v}$ & 70 & 65 & 60 & 40 & 80 \\
\hline & & & & & & & & & & & & & & & & & & & & $c_{v}$ & 2 & 3 & 6 & 2 & 4 \\
\hline
\end{tabular}

\section{Conclusion and further study}

Several natural disasters and catastrophes in the last decade convince researchers to find more effective ways to supplement those people who remain without water, food and somewhere to stay, and the people who require urgent medical treatments. In such circumstances, roads are influenced by disaster and quickly get out of the normal state. Therefore, some of them are not reliable more and rescue team should choose the routes with higher reliability level. For this purpose, we proposed a VRP model that maximizes the reliability by using an MADM method and the concept of permanent that considers significant factors that affect reliability of roads such as geographic characteristics, mountainous level of the road, road type (autobahn, arterial, lane, highway, etc.) and length. Moreover, the model is minimizing the total cost of vehicles and the unit load portage cost on the roads. The vehicles are heterogeneous with different capacity levels. One small-sized problem and one large-sized are solved. The first one is solved by using GAMS 24.0.2 software. Then for solving the problem in large size the NSGAII is investigated.

For further studies, several goals could be considered such as maximizing the satisfied demand or minimizing total time. In addition, the permanent concept could be extended into model and combined with other parameters like distance to obtain more realistic mathematical model. 


\section{References}

Afshar, A., \& Haghani, A. (2012). Modeling integrated supply chain logistics in real-time large-scale disaster relief operations. Socio-Economic Planning Sciences Journal, 46, 327-338.

Altay, N., \& Green, W.G. (2006). OR/MS research in disaster operations management. European Journal of Operational Research, 175, 475-493.

Barbarosoglu, G., Özdamar, L., \& Çevik, A. (2002). An interactive approach for hierarchical analysis of helicopter logistics in disaster relief operations. European Journal of Operational Research, 140, 118-33.

Baykasoglu, A. (2012). A review and analysis of graph theoretical-matrix permanent: approach to decision making with example applications. Springer Science and business media Dordrecht, 10, 1007-10462.

Bowers, J., Lyons, B., \& Mould, G. (2012). Developing a resource allocation model for the Scottish patient transport service. Operations Research for Health Care, 1, 84-94.

Bozorgi-Amiri, A., Jabalameli, M.S., Alinaghian, M., \& Heydari, M. (2012). A modified particle swarm optimization for disaster relief logistics under uncertain environment. International Journal of Advanced Manufacturing Technology, 60, 357-371.

Choi, M., \& Lee, S.H. (2012). The multiple traveling purchaser problem for maximizing system's reliability with budget constraints. Expert Systems with Applications, 38, 9848-9853.

Gulczynski, D., Golden, B., \& Wasil, E. (2011). The multi-depot split delivery vehicle routing problem: An integer programming-based heuristic, new test problems, and computational results. Computers \& Industrial Engineering, 61, 794-804.

Hamedi, M., Haghani, A., \& Yang, S. (2012). Reliable Transportation of Humanitarian Supplies in Disaster Response: Model and Heuristic. Procedia- Social and Behavioral Sciences, 54, 1205-1219.

Ho, W., T.S. Ho, G., Ji, P.C.W., \& Lau, H. (2008). A hybrid genetic algorithm for the multi-depot vehicle routing problem. Engineering Applications of Artificial Intelligence, 21, 548-557.

Huang, M., Smilowtiz, R., \& Balcik, B. (2013). A continuous approximation approach for assessment routing in disaster relief. Transportation Research Part B, 50, 20-41.

Huang, M., Smilowtiz, K., \& Backlit, B. (2012). Models for relief routing: Equity, efficiency and efficacy. Transportation Research Part E, 48, 2-18.

Jotshi, A., Gong, Q., \& Batta, R. (2009). Dispatching and routing of emergency vehicles in disaster mitigation using data fusion. Socio-Economic Planning Sciences, 43, 1-24.

Knott, R. (1987). The logistics of bulk relief supplies. Disasters, 11, 113-5.

Lee, S. (2012). The role of centrality in ambulance dispatching. Decision Support Systems, 54, 282-291.

Lin, Y.H., Batta, R., Rogerson, P.A., Blatt, A., \& Flanigan, M. (2011). A logistics model for emergency supply of critical items in the aftermath of a disaster. Socio-Economic Planning Sciences, 45, 132-145.

Mete H, \& Zabinsky Z. (2010). Stochastic optimization of medical supply location and distribution in disaster management. International Journal of Production Economics, 126, 76-84.

Miri Lavasani, M.R., Wang, J., Yang, Z., \& Finlay, J. (2012). Application of MADM in a fuzzy environment for selecting the best barrier for offshore wells. Expert Systems with Applications, 39, 2466-2478.

Özdamar, L., \& Demir, O. (2012). A hierarchical clustering and routing procedure for large scale disaster relief logistics planning. Transportation Research Part E, 28, 591-602.

Rath, S., \& Gutjahr, W.J. (2013). A math-heuristic for the warehouse location-routing problem in disaster relief. Computers \& Operations Research, 42, 25-39.

Sheu, J.B. (2010). Dynamic relief-demand management for emergency logistics operations under large-scale disasters. Transportation Research Part E, 46, 1-17.

Torre, L.E., Dolinskaya, I.S., \& Smilowitz, K. (2012). Disaster relief routing: Integrating research and practice. Socio-Economic Planning Sciences Journal, 46, 88-97.

Ukkusuri, S.V., \& Yushimito, WF. (2008). Location routing approach for the humanitarian prepositioning problem. Transportation Research Record: Journal of the Transportation Research Board, 2089, 18-25.

Vitoriano, B, Ortuno, T, \& Tirado, G. (2009). HADS, a goal programming-based humanitarian aid distribution system. Journal of Multi-Criteria Decision Analysis, 16, 55-64.

Widener, M.J., \& Horner, M.W. (2011). A hierarchical approach to modeling hurricane disaster relief goods distribution. Journal of Transport Geography, 19, 821-828.

Wohlgemuth, S., Oloruntoba, R., \& Clausen, U. (2012). Dynamic vehicle routing with anticipation in disaster relief. Socio-Economic Planning Sciences, 46, 261-271.

Yi, W., \& Kumar, A. (2007). Ant colony optimization for disaster relief operations. Transportation Research Part $E, 43,660-672$.

Zhang, J., Li, J., \& Liu, Z. (2012). Multiple-resource and multiple-depot emergency response problem considering secondary disasters. Expert Systems with Applications, 39, 11066-11071. 